\title{
Risk of Seroma with Simultaneous Liposuction and Abdominoplasty and the Role of Progressive Tension Sutures
}

\author{
Umar Daraz Khan
}

Published online: 7 December 2007

(C) Springer Science+Business Media, LLC 2007

\section{Erratum to: Aesth Plast Surg}

\section{DOI 10.1007/s00266-007-9004-y}

In the online version of this article, the current figure 4 caption sections $b$ and $c$ are duplicated from figure 3 . It appears correctly below:

Fig. $4(\mathrm{a}-\mathrm{b})$ Preoperative anterior and lateral view of a 37-year-old woman after weight loss with redundant skin and a weak musculoaponeurotic system. (c-d) Postoperative anterior and lateral views after type 4 abdominoplasty without liposuction

In addition, the last line of the Conclusion section appears incorrectly. The correct sentence is as follows:

No clinical difference was observed in wound healing of the distal skin flaps between the two groups.

The online version of the original article can be found under doi: 10.1007/s00266-007-9004-y

U. D. Khan $(\bowtie)$

Belvedere Private Hospital, Knee Hill Abbeywood,

London SE2 0GD, UK

e-mail: mrumarkhan@aol.com 